\title{
First experience with Tolvaptan for the treatment of neonates and infants with capillary leak syndrome after cardiac surgery
}

Anne Kerling ${ }^{1}$, Okan Toka ${ }^{1}$, André Rüffer ${ }^{2}$, Hanna Müller ${ }^{3}$, Sheeraz Habash ${ }^{1}$, Christel Weiss ${ }^{4}$, Sven Dittrich ${ }^{1}$ and Julia Moosmann ${ }^{1 *}$ (D)

\begin{abstract}
Background: Postoperative fluid management in critically ill neonates and infants with capillary leak syndrome (CLS) and extensive volume overload after cardiac surgery on cardiopulmonary bypass is challenging. CLS is often resistant to conventional diuretic therapy, aggravating the course of weaning from invasive ventilation, increasing length of stay on ICU and morbidity and mortality.
\end{abstract}

Methods: Tolvaptan (TLV, vasopressin type 2 receptor antagonist) was used as an additive diuretic in neonates and infants with CLS after cardiac surgery. Retrospective analysis of 25 patients with CLS including preoperative and postoperative parameters was performed. Multivariate regression analysis was performed to identify predictors for TLV response.

Results: Multivariate analysis identified urinary output during $24 \mathrm{~h}$ after TLV administration and mean blood pressure (BP) on day 2 of TLV treatment as predictors for TLV response (AUC $=0.956$ ). Responder showed greater weight reduction $(p<0.0001)$, earlier weaning from ventilator during TLV $(p=0.0421)$ and shorter time in the ICU after TLV treatment $(p=0.0155)$. Serum sodium and serum osmolality increased significantly over time in all patients treated with TLV.

Conclusion: In neonates and infants with diuretic-refractory CLS after cardiac surgery, additional aquaretic therapy with TLV showed an increase in urinary output and reduction in bodyweight in patients classified as TLV responder. Increase in urinary output and mean BP on day 2 of treatment were strong predictors for TLV response.

\section{Introduction}

Regulation of volume and electrolyte homeostasis after cardiac surgery on cardiopulmonary bypass $(\mathrm{CPB})$ in newborns and infants with congenital heart defects (CHD) is challenging $[1,2]$. The use of $\mathrm{CPB}$ during open heart surgery is accompanied by an inflammatory response leading to capillary leak syndrome (CLS) [3-5]. CLS can be defined by the clinical presentation of third space volume overload with consecutive generalized edema and substantial gain of weight, intravascular hypovolemia,

\footnotetext{
* Correspondence: julia.moosmann@uk-erlangen.de

${ }^{1}$ Department of Pediatric Cardiology, University of Erlangen-Nürnberg,

Loschgestrasse 15, 91054 Erlangen, Germany

Full list of author information is available at the end of the article
}

hypoalbuminemia and hemoconcentration in the absence of severe congestive heart failure (CHF). In conjunction, an elevation of subcutaneous-thoracic ratio (ST-ratio) can help to diagnose CLS in the pediatric population $[3,4,6$, 7]. Prolonged interstitial fluid retention due to CLS is often resistant to conventional diuretic therapy, aggravating weaning from invasive ventilation, leading to longer time at the ICU and increasing postoperative morbidity (e.g. pulmonary infections) and mortality $[4,8,9]$. There have been great efforts in early detection and prevention of CLS $[3,10]$. However, improvements in treatment strategies especially for neonates and children after cardiac surgery are still needed.

(c) The Author(s). 2019 Open Access This article is distributed under the terms of the Creative Commons Attribution 4.0 International License (http://creativecommons.org/licenses/by/4.0/), which permits unrestricted use, distribution, and reproduction in any medium, provided you give appropriate credit to the original author(s) and the source, provide a link to the Creative Commons license, and indicate if changes were made. The Creative Commons Public Domain Dedication waiver (http://creativecommons.org/publicdomain/zero/1.0/) applies to the data made available in this article, unless otherwise stated. 
Tolvaptan (TLV) is a selective competitive vasopressin 2 receptor antagonist and prohibits the movement of aquaporin 2 into the luminal wall of the collecting duct and thereby reduces the reabsorption of water [11, 12]. TLV has been FDA (Food and Drug Administration) approved for the treatment of hyponatremia associated with $\mathrm{CHF}$ in adults and the syndrome of inappropriate antidiuretic hormone secretion (SIADH) in adults and children. The approval for treatment of hyponatremia in patients with liver cirrhosis was removed due to reported hepatotoxicity in adults, and the duration of treatment was limited to 30 days. TLV has also shown efficacy in treatment of autosomal-dominant polycystic kidney disease [12-16].

Several studies including a phase III study illustrated the efficacy of TLV in CHF with hypervolemia and hyponatremia especially during the acute phase of cardiac decompensation and diuretic resistance in adults [17-19]. The multicenter, retrospective J-SPECH study from 2015 suggested that TLV can be safely administered in pediatric patients but may be less effective in neonates and infants compared to adolescence or adults [14,20]. Differences in the response profiles to TLV were often seen, however they had been unpredictable in the beginning. Recent studies defined TLV response as an increase of urine volume after its administration, patients responding with an increase are defined as responder [21,22].

The role of TLV in postoperative fluid management after cardiac surgery on CPB has been evaluated in postoperative treatment in adults, but little is known about its role in infants and neonates $[18,23,24]$. One recent retrospective study in pediatric patients after uncomplicated cardiovascular surgery (shunt closure) compared treatment of additional TLV to patients treated with standard diuretic therapy [25]. TLV treatment was safely administered and resulted in an increase in urinary output, showing a potential reduction of intravenous loop-diuretic use during treatment course [25].

We used TLV in the postoperative fluid management in critically ill infants and neonates with postoperative CLS, massive volume overload and diuretic resistance after complex cardiac surgery and we retrospectively analyzed parameters to predict TLV response.

\section{Materials and methods \\ Patients}

Our retrospective analysis encompasses a single center experience (Department of Pediatric Cardiology at the Friedrich-Alexander-University of Erlangen-Nürnberg, Germany). We included 25 patients with CHD after cardiac surgery, treated with TLV in ICU between June 2011 and May 2017, evaluating effects of postoperative TLV therapy in patients with CLS. Criteria for the use of add-on therapy was 1) fluid overload 2) no increase in urinary output under conventional diuretic therapy 3) persisting renal function (no anuria) 4) low serum sodium. Descriptive patient's auxologic and clinical characteristics, leading cardiologic diagnosis and the respective surgical procedures are displayed in Tables 1 and 2. Our cohort included four preterm patients $(1: 31+6 ; 2: 35+$ 6; $3: 34+5 ; 4: 34+5)$. Corrected age for preterm infants at TLV treatment was $35+3,39+5,41+0$ and one infant received therapy 8 month after birth.

We evaluated Risk Adjusted Congenital Heart Surgery score (RACHS-1) [26, 27] and basic Aristotle score [28] to quantify risk and complexity of the performed surgeries. STS-EACTS mortality category and associated major complications $(95 \% \mathrm{CrI})$ were implemented to express mortality associated with congenital heart surgery and classifying congenital heart surgery procedures on the basis of their potential for morbidity [29, 30]. Team of surgeons, anesthesiologists and pediatric cardiologists remained unchanged during the study period. All patients underwent median sternotomy. Post-operative treatment was exclusively supervised on the pediatric cardiology ICU, beat-to-beat circulatory and pulmonary status, fluid and electrolyte homeostasis was digitally monitored, clinical status and organ function was monitored and digitally documented routinely by critical care nursing staff. Co-medication including conventional diuretic therapy before and during the treatment course with TLV was analyzed.

\section{Definition of CLS, responder- and non-responder - grouping}

CLS was defined by clinical symptoms (volume overload, intravascular hypovolemia, low total protein, hypoalbuminemia, hemoconcentration) and subcutaneous-thoracic ratio (ST-Ratio; > 97. percentile). ST-Ratio was evaluated to quantify CLS by chest $\mathrm{x}$-ray with anterior-posterior beam path [6]. X-rays were documented with Web Ris (celsius37.com AG, Mannheim, Germany).

Responder to TLV were classified according to the definition from adult studies by Imamura et al. [21, 22, 31] "responders as patients with any increase in urine volume (UV) at day 1 when TLV administration was started". To classify individuals as responder in our population to TLV we permitted an increase of $>10 \%$ in urinary output within $24 \mathrm{~h}$ after the first TLV administration. Others were classified as non-responder [20-22].

\section{Treatment protocol}

TLV ("Samsca", Otsuka, Japan) was administered as individual healing attempt in critically ill children with complicated postoperative course. Off-label use was explained and informed consent was obtained by all participating families. Starting dose of TLV was $25 \%$ of target dose $(1 \mathrm{mg} / \mathrm{kg} / \mathrm{d})$. Dose finding was titrated based on 
Table 1 Patient demographics

\begin{tabular}{|c|c|c|c|}
\hline \multirow[t]{2}{*}{ Parameter } & \multicolumn{2}{|l|}{$\mathrm{CLS}$} & \multirow{2}{*}{$\begin{array}{l}P \text { - } \\
\text { value }\end{array}$} \\
\hline & Responder & Non-responder & \\
\hline$n$ & 17 & 8 & \\
\hline Female & 9 & 4 & 1.0000 \\
\hline Male & 8 & 4 & \\
\hline Gestagional age (SSW) & $37+4(31+6-40+1)$ & $38+1(37+1-40+0)$ & 0.1049 \\
\hline RACHS-1score & $3(2-6)$ & $3(2-6)$ & 0.2253 \\
\hline Basic Aristotle score & $10(4-15)$ & $7.25(6.5-14.5)$ & 0.0997 \\
\hline Cardiopulmonary bypass time (min) & $219(0-390)$ & $148(56-439)$ & 0.1709 \\
\hline Cross clamp time (min) & $77(0-177)$ & $51(8-173)$ & 0.3508 \\
\hline Subcutaneous-thoracic-ratio (\%) & $21.0(14.3-26.5)$ & $19.75(14.7-27.9)$ & 0.6408 \\
\hline Secondary chest closure after surgery (days) & $8(2-24)(n=11)$ & $10(2-17)(n=4)$ & 0.9878 \\
\hline Start of TLV after surgery (days) & $13(2-44)$ & $15(7-24)$ & 1.0000 \\
\hline Age when TLV therapy was started (days) & $35(9-228)$ & $37.5(20-549)$ & 0.3821 \\
\hline Preoperative weight (kg) & $3.30(1.88-4.27)$ & $3.20(1.93-6.32)$ & 0.7487 \\
\hline Absolute weight before TLV (kg) & $4.35(2.83-5.55)$ & $4.42(2.61-5.68)$ & 0.8673 \\
\hline Weight above dry weight when TLV was started (\%) & $131.8(102.6-202.8)$ & $133.5(113.5-154.4)$ & 0.8151 \\
\hline Dose of TLV administration (mg/kg) & $0.53(0.15-1.06)$ & $0.49(0.13-0.95)$ & 0.6204 \\
\hline Period of TLV administration (days) & $8(1-25)$ & $7(1-47)$ & 0.6391 \\
\hline Urinary output $24 \mathrm{~h}$ prior to Tolvaptan administration $(\mathrm{ml} / \mathrm{kg} / \mathrm{h})$ & $4.15(0.92-9.18)$ & $3.27(0.54-9.40)$ & 0.4665 \\
\hline Urinary output $24 \mathrm{~h}$ after Tolvaptan administration (ml/kg/h) & $6.38(1.20-15.41)$ & $2.21(0.28-7.15)$ & 0.0039 \\
\hline Days on ICU after TLV administration & $15(3-111)$ & $40.5(16-139)$ & 0.0155 \\
\hline Death & 1 & 3 & 0.0808 \\
\hline
\end{tabular}

Frequencies are given for binary data; for quantitative and ordinal data median and range are presented. $p<0.05$ has been considered as statistically significant

clinical symptoms, side effects (see below) and serum sodium levels. Tablets are available in $15 \mathrm{mg}$ and $30 \mathrm{mg}$. Provision of small dosages was performed by the department of pharmacology of the University hospital Erlangen. Tablets were pulverized and encapsulated. At the ICU the pulverized aliquots were diluted and administered via nasogastric tube.

\section{Definition of TLV related adverse events}

Adverse events were retrospectively analyzed according to the criteria of Otsuka applying for the planned Phase $3 \mathrm{~b}$, multicenter study trial "effects of TLV in hospitalized children with euvolemic or hypervolemic serum hyponatremia".

Adverse events are classified: 1) absolute serum sodium level $>145 \mathrm{mmol} / \mathrm{L}$ or an overly rapid rise in serum sodium level (an increase in serum sodium of $>8 \mathrm{mmol} /$ $\mathrm{L}$ over a 10 -h period, $12 \mathrm{mmol} / \mathrm{L}$ over a $24-\mathrm{h}$ period. 2 ) neurological symptoms, or other signs or symptoms suggestive of osmotic demyelination. 3) worsening symptoms of hyponatremia. 4) elevations in AST or ALT that are $>2 \times$ ULN (upper limit of normal) or levels that increase $>2$ times their previously observed level.

\section{Data collection}

$\mathrm{TLV}$ doses were calculated in $\mathrm{mg} / \mathrm{kg}$ (preoperative weight)/d. Volume overload was quantified, assuming preoperative weight as $100 \%$. TLV application period, time on mechanical ventilation, time until extubation, body weight, urinary output and total daily dose of selected concurrent medications were recorded by Integrated Care Manager (ICM, Drägerwerk AG \& Co. KGaA, Lübeck, Germany) software solutions. Retrospective data acquisition of laboratory values before surgery, before TLV treatment and during TLV treatment was performed using Lauris (version 15.09.29.9, Swisslab GmbH, Berlin, Germany) (Table 1).

\section{Institutional protocol for transfusion and fluid management}

Post-operative indication for transfusion was alike and followed our departmental transfusion algorithm: packed red blood cells (PRBC) were administered at a 
Table 2 Diagnosis and surgical procedures

\begin{tabular}{|c|c|c|c|c|c|}
\hline Diagnosis & Operation & Responder & $\begin{array}{l}\text { Non- } \\
\text { responder }\end{array}$ & $\begin{array}{l}\text { STS-EACTS mortality } \\
\text { category }\end{array}$ & $\begin{array}{l}\text { Major complications } \\
(95 \% \mathrm{Crl})\end{array}$ \\
\hline Aortic arch hypoplasia & Reconstruction of the aortic arch & 1 & & 3 & $12.2 \%$ \\
\hline \multirow[t]{2}{*}{$\begin{array}{l}\text { Dextro Transposition of the great } \\
\text { arteries ( } d \text {-TGA) }\end{array}$} & $\begin{array}{l}\text { Arterial switch operation + ASD and/or } \\
\text { VSD closure }\end{array}$ & 4 & & 3 & $10.7 \%$ \\
\hline & $\begin{array}{l}\text { Arterial switch operation + VSD patch } \\
\text { and aortic arch repair }\end{array}$ & 1 & & 5 & $31.0 \%$ \\
\hline Pulmonary atresia & RVOT patch- enlargement & & 1 & 2 & $6.5 \%$ \\
\hline \multirow[t]{4}{*}{ Single ventricle } & Norwood & 2 & & 5 & $29.7 \%$ \\
\hline & Bidirectional Glenn-anastomosis & 1 & 1 & 2 & $6.4 \%$ \\
\hline & aortic arch reconstruction & 1 & 1 & 4 & $15.9 \%$ \\
\hline & $\begin{array}{l}\text { DKS anastomosis + aortic arch } \\
\text { reconstruction }\end{array}$ & 1 & & 5 & $22.9 \%$ \\
\hline \multirow[t]{2}{*}{ Fallot-Tetralogy (TOF) } & TOF repair (RVOT patch, VSD patch) & 1 & 1 & 1 & $7.7 \%$ \\
\hline & Blalock-Taussig-Shunt & 1 & & 4 & $12.4 \%$ \\
\hline Interrupted aortic arch & aortic arch repair & 1 & 1 & 4 & $19.0 \%$ \\
\hline \multirow[t]{2}{*}{ Double outlet right ventricle (DORV) } & Closure of aorto-pulmonary-window & 1 & & 2 & $6.2 \%$ \\
\hline & Norwood & 1 & & 5 & $29.7 \%$ \\
\hline Tricuspid atresia lb & Aorto-pulmonary shunt & & 1 & 4 & $11.3 \%$ \\
\hline $\begin{array}{l}\text { Total anomalous pulmonary venous } \\
\text { return (TAPVC) }\end{array}$ & Correction of pulmonary vein anomalies & 1 & 1 & 4 & $16.4 \%$ \\
\hline Mitral valve insufficiency & $\begin{array}{l}\text { Mitral valve reconstruction, Ring } \\
\text { implantation }\end{array}$ & & 1 & 2 & $6.6 \%$ \\
\hline Total & & 17 & 8 & & \\
\hline Mean & & & & 3.4 & $15.3 \%$ \\
\hline Responder & & & & 4 & $12.4 \%$ \\
\hline Non responder & & & & 3 & $9.5 \%$ \\
\hline
\end{tabular}

Values are expressed as absolute frequencies for binary data

hemoglobin $(\mathrm{Hb})$ level of $14 \mathrm{~g} / \mathrm{dl}$ in cyanotic patients and $10 \mathrm{~g} / \mathrm{dl}$ in non-cyanotic patients. In the case of on-going bleeding, fresh frozen plasma (FFP, 10-15 ml/ $\mathrm{kg}$ ) was transfused if quick reached below $50 \%$. Platelets were transfused at a platelet count below $50 \times 10^{3} / \mu \mathrm{l}$.

Postoperative indication for fluid substitution of kristalloids $(\mathrm{NaCl}$ and Jonosteril) is central venous pressure $(\mathrm{CVP})<5$, and low blood pressure $(\mathrm{BP})$ according to age related reference ranges. Administration of colloidal volume expanders, i.e. albumin and hydroxyethyl starch (HAES) is performed in hemodynamically unstable cases or low serum albumin levels.

\section{Statistical analysis}

Quantitative approximately normally distributed variables are expressed as mean \pm standard deviation (SD). For ordinally scaled data (e.g. RACHS-1) and for variables with skewed distribution median value together with minimum and maximum are given. As most of variables in Table 1 (demographic parameters, co-medication and laboratory parameters) and 3 (co-medication and laboratory parameters) seem to be normally distributed and due to the rather small sample sizes non-parametric Mann-Whitney-U tests have been performed in order to compare the median values of the two groups. For qualitative factors (i.e. cardiac malformation or procedures) absolute frequencies are presented. Fisher's exact tests have been used.

In order to investigate changes over time (regarding weight, serum sodium, osmolality, and urinary output) ANOVAs for repeated measurements have been performed including time point and responder group as fixed factors and patients' ID as a random factor. For the liver enzymes, Friedman's test was performed instead of ANOVA for repeated measurements, because of the skewed distribution. Multiple regression analysis including all parameters was performed to identify predictive parameters for TLV responder. 
All statistical analyses were conducted using GraphPad Prism (version 6.05, GraphPad Software, Inc., La Jolla, CA 92037 USA) and SAS, release 9.4 (SAS institute Inc., Cary. NC, USA). The result of a statistical test has been considered as statistically significant if the $p$ value was less than 0.05 .

\section{Ethical statement}

The retrospective study was approved by the ethics committee of the University of Erlangen-Nürnberg (Re.-No. 145_13B). The study was conducted in accordance with the Declaration of Helsinki [32].

\section{Results}

\section{Demographics}

Postoperative CLS was diagnosed in 25 patients after cardiac surgery. Clinical parameters to define CLS are displayed in Table 1.

According to the definition of TLV responder by Imamura et al. [21, 22, 31] 17 individuals were identified as responder to TLV defined by an increase in urinary output $>10 \%$ in $24 \mathrm{~h}$ and 8 infants were identified as non-responder [20-22] (Table 1).

Age was similar in both groups (median 35 and 37.5 days; $p=0.3821$ ). The underlying cardiac malformation and surgical procedures are displayed in Table 2. Extracardiac malformations and syndromes were Trisomy 21 in one responder and one non-responder patient, Turner syndrome in one non-responder and omphalocele in one responder patient. Surgical parameters (cardio pulmonary bypass $(\mathrm{CPB})$ time, cross clamp time and surgical risk scores RACHS-1 and Aristotele score) are displayed in Table 1. In 15 patients, primary chest closure was not possible and secondary closure was performed. Both groups presented with increased ST-ratio $>97$. percentile $(p=0.6408)$. A significant positive correlation was identified between ST-ratio and time on CPB $(p=0.0305$, Pearson's correlation coefficient $r=0.4333$ ). STS-EACTS mortality category was 4 in responder and 3 in non-responder $(p=0.2201)$ and estimated major complication rates are $15.3 \%$ in responder compared to $12.4 \%$ in non-responder $(p=0.2190)$. Four responder patients showed severe infection with elevated procalcitonin (PCT) ( $n=1$ necroticing enterocolitis, $n=1$ positive blood culture with Straphylococcus epidermidis, $n=1$ pneumonia with Enterococcus faecalis, $n=1$ Enterococcus faecium wound infection). Infection rates normalized before TLV treatment in all responder patients. One non-responder patient presented with an infection during treatment ( $n=1$ Staphylococcus epidermidis in intraoperative pericardial swab). Postoperative major complications are demonstrated in Table 3.

Postoperative days on ICU, before TLV therapy was started $(p=1.0000)$, preoperative weight $(p=0.7487)$ and absolute weight $(p=0.8673)$ when TLV was started were not significantly different between responder and non-responder. All individuals presented with increased body-weight with a median of $131.8 \%$ over their preoperative weight in the responder group and $133.5 \%$ in the non-responder group, when TLV was started $(p=0.8151)$. The duration of TLV application $(p=0.6391)$ and average dose of TLV $(p=0.6204)$ administered were similar. Median length of stay in the ICU after TLV administration was significantly shorter in responder compared to non-responder patients ( 15 vs. 40.5 days; $p=0.0155$ ).

We observed four deaths in the study population, one responder and three non-responder $(p=0.0808$.) 17 days, 34 days, 35 days and 48 days after starting TLV.

Laboratory parameters were analyzed at several time points. Preoperative parameters did not show significant differences between both groups (Additional file 1: Table S1). Before TLV treatment non-responder group presented with a higher hematocrit $(p=0.0169)$ and higher hemoglobin level $(p=0.0168)$. According to CLS criteria: total protein was lowered in both groups (responder: $37.0 \mathrm{~g} / \mathrm{l}$ and non-responder: 38.84 $\mathrm{g} / \mathrm{l} ; p=0.9303)$ and median albumin levels were decreased in responder $20.15 \mathrm{~g} / \mathrm{l}$ and non-responder

Table 3 Major complications

\begin{tabular}{|c|c|c|c|}
\hline & Responder & Non-Responder & $p$-value \\
\hline \multicolumn{4}{|c|}{ Postoperative acute renal failure requiring temporary dialysis } \\
\hline Hemodialysis & 1/17 (10 days) & 1/8 (18 days) & 1.0000 \\
\hline PD & 6/17 (10 days; 3-19) & 6/8 (10 days; 4-29) & $0.1936 / 0.8099$ \\
\hline Postoperative neurologic deficit & $1 / 17$ & $1 / 8$ & 1.0000 \\
\hline Postoperative mechanical circulatory support & 5/17 (8 days; 4-12) & 5/8 (12 days; 7-34) & $0.1936 / 0.2073$ \\
\hline Phrenic nerve injury & $3 / 17$ & $1 / 8$ & 1.0000 \\
\hline Unplanned reoperation & $2 / 17$ & $3 / 8$ & 0.2833 \\
\hline
\end{tabular}

Major complications according to the Society of Thoracic Surgeons. Values are expressed as median and range. $p<0.05$ has been considered as statistically significant.Fisher-Test was used to compare numbers of complications in both groups, Mann-Whitney-U-Test was used for differences between durations 
Table 4 Co medication and laboratory parameters

\begin{tabular}{|c|c|c|c|}
\hline & Responder & Non responder & $P$-value \\
\hline \multicolumn{4}{|c|}{ Co-medication (mg/kg/day) when TLV was started } \\
\hline Furosemide perfusor & $5.93(0.60-7.98)(n=16)$ & $5.57(1.70-10.9)(n=8)$ & 0.1522 \\
\hline Thiazide oral & $1.93(0.99-3.72)(n=12)$ & $1.03(0.46-2.09)(n=5)$ & 0.1703 \\
\hline Spironolactone oral & $2.27(0.93-6.38)(n=13)$ & $2.08(2.05-2.78)(n=4)$ & 0.7339 \\
\hline Etacrynacid intravenous & $1.09(0.69-3.41)(n=5)$ & $1.15(1.04-1.26)(n=2)$ & 1.0000 \\
\hline \multicolumn{4}{|c|}{ Laboratory parameters before TLV treatment } \\
\hline Hematocrit (\%) & $40.07(31.73-53.60)$ & $44.08(39.87-51.37)$ & 0.0169 \\
\hline Hemoglobin (g/dl) & $13.12(9.5-19.02)(n=16)$ & $14.25(13.40-16.83)$ & 0.0168 \\
\hline Total protein $(\mathrm{g} / \mathrm{l})$ & $37.0(27-45.67)$ & $38.84(31.67-44.00)$ & 0.9303 \\
\hline Albumin (g/l) & $20.15(18.43-26.40)(n=7)$ & $21.50(17.8-25.0)(n=7)$ & 0.7983 \\
\hline serum BUN (mg/dl) & $42.00(7.67-113.50)$ & $50.83(24.33-109.17)$ & 0.4316 \\
\hline Creatinine (mg/dl) & $0.44(0.24-1.47)$ & $0.66(0.25-1.02)$ & 0.4484 \\
\hline Sodium (mmol/l) & $135(129-144)$ & $130.5(126.17-137.67)(n=7)$ & 0.1269 \\
\hline Potassium (mmol/l) & $4.10(3.63-4.80)$ & $4.09(3.80-4.60)$ & 0.8156 \\
\hline Osmolality (mosm/kg) & $281.67(267.5-307.0)(n=16)$ & $280.92(261.0-291.0)$ & 0.4258 \\
\hline
\end{tabular}

For quantitative and ordinal data median and range are presented. $p<0.05$ has been considered as statistically significant

$21.50 \mathrm{~g} / \mathrm{l}(p=0.7983)$. Serum sodium levels were low/ normal in both groups (responder: $135 \mathrm{mmol} / \mathrm{l}$ vs. $130.5 \mathrm{mmol} / \mathrm{l} ; \quad p=0.1269)$. No differences were observed for serum blood urea nitrogen (BUN), creatinine, potassium and serum osmolality before TLV treatment was started (Table 4).

Vital parameters including (BP, heart rate (HR) and CVP) were analyzed. CVP decreased during TLV treatment in both groups, but was not significantly different. Mean BP was lower in non-responder on day $2(p=0.0035)$ and day $3(p=0.0309)$ of treatment (Additional file 1: Table S2).

\section{Predicting TLV response}

Multivariate regression analysis to predict TLV response revealed mean BP on day 2 of TLV administration and urinary output $24 \mathrm{~h}$ after TLV as significant combined predictors for responder to TLV. Predicting TLV response with an AUC $=0.956$.

The probability for TLV response increases by 1.185 / mmHg mean BP on day 2 of TLV treatment and the probability for TLV response increases by factor $2.064 / \mathrm{ml} / \mathrm{kg} /$ $\mathrm{h}$ urinary output after $24 \mathrm{~h}$ after TLV administration.

Mathematical model to estimate the probability for responder:
Tolvaptan effects on bodyweight, serum sodium levels, osmolality and urinary output

For each parameter (bodyweight, serum sodium, osmolality and urinary output) and for each group (responders, non-responders) changes over time could be observed (with the only exception for the weight parameter in the non-responder group) (Fig. 1a-d).

Responders showed a significant weight reduction starting at day \# 2 after TLV administration. The greatest weight reduction was achieved at day \# 7 of treatment down to $115.6 \pm 7.1 \%(p<0.0001)$ of preoperative weight. Fig. 1a shows the weight progression between responder and non-responder group over 10 days of TLV administration. Non-responder did not show a significant weight reduction in the investigated time period $(p=0.1067)$, while responders showed a significant weight reduction $(p<0.0001)$ (Fig. 1).

Urinary output $24 \mathrm{~h}$ after the first dose of TLV was significantly higher (by definition of responder) in the responder group ( $p=0.0039$; Table 1; Fig. 1d). During all 10 days of treatment urinary output stayed higher (related to day 0) in the responder group. In the non-responder group urinary output also increased over the total investigated time period $(p=$ $0.0003)$, but a significant increase from day \# 0 was

probability for response to TLV $=\frac{\exp (-12.34+0.1696 \times \text { mean bp day } 2 \text { of } T L V+0.7248 \times \text { " urinary output } 24 h \text { after TLV })}{1-(\exp (-12.34+0.1696 \times \text { mean bp day } 2 \text { of } T L V+0.7248 \times \text { "urinary output } 24 h \text { after TLV }))}$ 

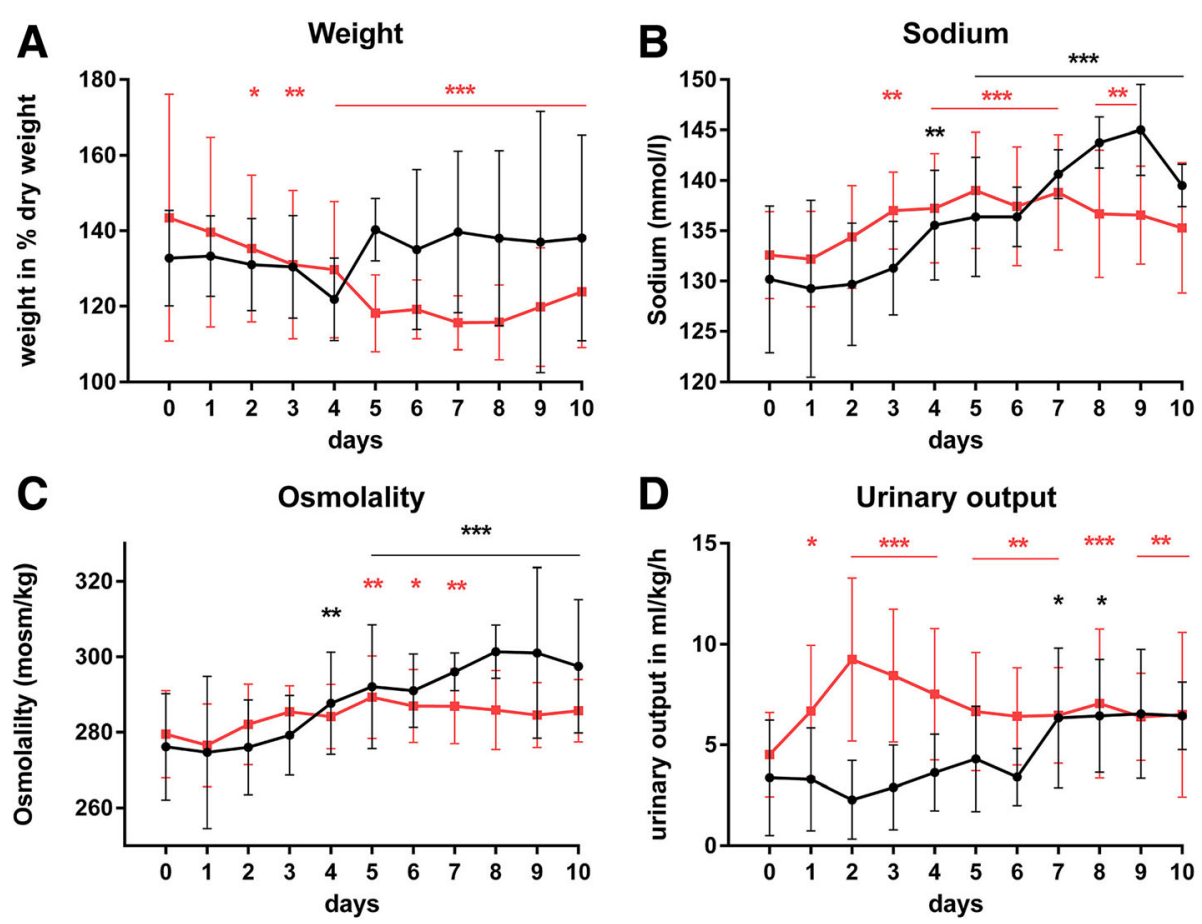

$\rightarrow$ Non-Responder

$\rightarrow$ Responder

\begin{tabular}{|c|c|c|c|c|c|}
\hline Parameter & Group effect & Interaction & Time effect & $\begin{array}{c}\text { Time effect } \\
\text { responder }\end{array}$ & $\begin{array}{c}\text { Time effect } \\
\text { non-responder }\end{array}$ \\
\hline Weight & $\mathrm{p}=0.576$ & $\mathrm{p}<0.0001$ & $\mathrm{p}=0.148$ & $\mathrm{p}<0.0001$ & $\mathrm{p}=0.107$ \\
\hline Serum sodium & $\mathrm{p}=0.549$ & $\mathrm{p}<0.0001$ & $\mathrm{p}<0.0001$ & $\mathrm{p}<0.0001$ & $\mathrm{p}<0.0001$ \\
\hline Serum osmolality & $\mathrm{p}=0.065$ & $\mathrm{p}<0.0001$ & $\mathrm{p}<0.0001$ & $\mathrm{p}=0.001$ & $\mathrm{p}<0.0001$ \\
\hline Urinary output & $\mathrm{p}=0.007$ & $\mathrm{p}=0.0001$ & $\mathrm{p}=0.006$ & $\mathrm{p}<0.0001$ & $\mathrm{p}=0.0003$ \\
\hline
\end{tabular}

Fig. 1 Weight (a), serum sodium (b), serum osmolality (c) and urinary output (d) during 10 days of TLV treatment. Responders (red graph) and non-responder (black graph); ${ }^{*} p<0.05$ (related to day 0 ) ${ }^{* *} p<0.01$ (related to day 0 ) ${ }^{* *} p<0.001$ (related to day 0 ). $p$-values deriving from 2 way ANOVAs; $p$ values for time effect deriving from 2 separate ANOVAS for responders and non-responders. Changes over time regarding bodyweight, serum sodium, osmolality and urinary output have been tested using ANOVAs for repeated measurements with group (responder / non-responder) and time point as fixed factors. The p-values in Table 2 reveal that for each parameter interactions between group and time effects could be observed indicating that response profiles of the two groups differ (see Fig. 1a and d)

later than in the responder group on day 7 and 8 of treatment (Fig. 1d).

Before TLV therapy, responder and non-responder presented with median serum sodium at the lower cut off to normal. A significant increase was identified during the investigated time period in both groups $(p<0.0001)$ (Fig. 1b). No significant difference between responder and non-responder groups was observed ( $p=0.5489$, accumulated over time), however the response profiles were different $(p<0.0001)$. In responder, a significant increase of serum sodium was seen at day \# 3 , in non-responder at day \# 4. In the responder group, hypernatremia was not observed. We observed one adverse event related to TLV in the non-responder group, one patient developed hypernatremia $(151 \mathrm{mmol} / \mathrm{l})$ on day \# 9, which was reversible on the following day.

Osmolality increased in both groups over treatment course (non-responder $p<0.0001$ and responder $p=$ 0.001) (Fig. 1c). Significant changes in osmolality were seen on day \# 4 in the non-responder and on day \# 5 in the responder group (Fig. 1c).

\section{Liver metabolism}

Liver enzymes were monitored before, during and after TLV treatment course. Due to the limitations of retrospective data analysis measurements were not performed on a regular basis of a distinct study protocol. Regarding the 
upper cut off values of alanine- aminotransferase (ALT; normal $<26 \mathrm{U} / \mathrm{l}$ ), aspartate- aminotransferase (AST; normal $<50 \mathrm{U} / \mathrm{l}$ ) and Gamma-Glutamyltransferase (GGT; normal $<23 \mathrm{U} / \mathrm{l}), 3 / 8$ of the responder, $4 / 8$ of the non-responder presented with significantly elevated GGT before TLV treatment, already. $2 / 8$ of non-responder presented with initial AST elevation. ALT elevation was present in $3 / 8$ of the non-responder. In both groups no significant elevation of AST, ALT and GGT was identified for median group parameters during and after treatment (Table 5).

\section{Co-medication, transfusions and fluid management}

Diuretic and catecholamine therapy before surgery is listed in Additional file 1: Table S1 presenting no differences between both groups. Postoperative catecholamine therapy and diuretic treatment before TLV administration was not different between responder and non-responder (Additional file 1: Table S3). Intravenous additional diuretic therapy could be reduced in both groups during treatment course with TLV (by factor 3.68 and 3.77, respectively). An ANOVA for repeated measurements revealed no statistical difference between the responders and non-responders $(p$ $=0.3935)$ and no statistically significant interaction term $(p$ $=0.6127)$. However, reduction over the investigated time could be observed in both groups $(p<0.0001)$. Nephrotoxic medication (i.e. vancomycin, fluconazole and tobramycin) was administered in a subset of patients in both groups, no differences were observed (Additional file 1: Table S3). Estimated glomerular filtration rate (GFR; by Schwartz formula) before and during treatment is provided in Table 6 . All patients received postoperative kristalloids, substitution during TLV and after TLV is listed in Additional file 1: Table S3 and did not show differences between both groups. Only a very limited number of patients received kolloids, mainly albumin. HAES was only substituted in two non-responder patient during the immediate postoperative course (Additional file 1: Table S3).

\section{Airway management}

Mechanical or non-invasive ventilation was required in all CLS patients (Table 6). All 17 responder patients needed mechanical ventilation before TLV administration. In 10 patients, invasive ventilation could be ended during TLV administration. In 2 responder patients, extubation was performed within 7 days after TLV. 3 individuals were extubated more than 7 days after TLV treatment course. 2 patients were not extubated and received a tracheostoma. In the non-responder group, 7 patients required mechanical ventilation, only one of them could be extubated during treatment course with TLV. Horovitz-index (oxygenation index) demonstrates improvements of respiration therapy and increased during treatment (Table 6). It was higher in responder compared to non-responder but did not show significant differences. Rate of extubation during TLV treatment was higher in responder compared to non-responder $(p$ $=0.0421$, Table 6).

\section{Discussion}

We report a retrospective analysis of our single center experience with TLV treatment in infants and neonates after cardiac surgery with postoperative CLS to predict TLV response. Additional diuretic therapy with TLV increased urinary output $>10 \%$ in $2 / 3$ of patients with CLS. According to the definition of Imamura et al. [21, $22,31]$ patients with increased urinary output during the first $24 \mathrm{~h}$, were classified as responder to TLV and presented with significant reduction in body weight. Increase in urinary output during the first $24 \mathrm{~h}$ after TLV administration and higher mean BP on day 2 of TLV treatment were identified as predictive factors for TLV response (AUC $=0.956)$.

The underlying mechanisms of TLV response have been studied in detail; TLV is able to antagonize antidiuretic hormone $(\mathrm{ADH})$ overstimulation and thus increases urinary output due to aquaresis. ADH excretion can be triggered by intravascular hypovolemia, activation of renin angiotensin aldosterone (RAAS) axis (mainly angiotensin II, by chronic extensive diuretic abuse), reduced osmotic pressure (plasma osmolality $<275 \mathrm{mmosm} / \mathrm{kg}$ ), stress and post-operative pain $[33,34]$. All these parameter can be observed in pediatric patients with CLS due to long CPB time, presenting with third space volume overload and intravascular volume depletion and therefore no severe hyponatremia but low to normal serum sodium levels. In contrast, patients with postoperative renal or cardiac failure presenting with volume

Table 5 Liver metabolism

\begin{tabular}{llllll}
\hline Group & Parameter & Before TLV & Under TLV & After TLV & $P$-value \\
\hline Responder $(n=17)$ & GGT $(<23 \mathrm{U} / \mathrm{l})$ & $41.5(19-93) n=8$ & $95.5(29-215) n=6$ & $118(38-354) n=9$ & 0.2926 \\
Non-responder $(n=8)$ & & $53.5(17-220)$ & $93(34-352) n=7$ & $116.5(24-845) n=6$ & 0.2223 \\
Responder $(n=17)$ & AST $(<50 \mathrm{U} / \mathrm{l})$ & $22.5(10-48) n=8$ & $20.5(10-57) n=8$ & $23.5(14-143) n=10$ & 0.4576 \\
Non-responder $(n=8)$ & & $32.5(11-638)$ & $57(14-252) n=6$ & $28.5(13-50)$ & 0.1677 \\
Responder $(n=17)$ & ALT $(<26 \mathrm{U} / \mathrm{l})$ & $16(9-30) n=7$ & $14(6-178) n=9$ & $17.5(10-26) n=8$ & 0.4987 \\
Non-responder $(n=8)$ & & $30.5(11-382)$ & $51(7-399) n=7$ & $21.5(6-107)$ & 0.1303 \\
\hline
\end{tabular}

Normal values for GGT, AST and ALT are expressed. Values are expressed as median and range. $p<0.05$ has been considered as statistically significant 
Table 6 Airway management and GFR

\begin{tabular}{|c|c|c|c|}
\hline & Responder & Non-responder & $p$-value \\
\hline Mechanical ventilation & 17 & 7 & 0.3200 \\
\hline Non-invasive ventilation & 0 & 1 & 0.3200 \\
\hline Extubation during TLV & 10 & 1 & 0.0421 \\
\hline Extubation 2-7 days after TLV & 2 & 0 & 1.0000 \\
\hline Extubation $>7$ days after TLV & 3 & 3 & 0.3442 \\
\hline No Extubation and tracheostoma & 2 & 3 & 0.2833 \\
\hline \multicolumn{4}{|l|}{ Oxygenation index } \\
\hline Postoperative & $173(45-365)$ & $137(38-253)$ & 0.0857 \\
\hline Before TLV & $235(127-366)$ & $139(83-284)$ & 0.0702 \\
\hline 1st day & $241(103-377)$ & $162(132-243)$ & 0.1829 \\
\hline 2nd day & $264(110-355)$ & $181(103-272)$ & 0.0524 \\
\hline 3rd day & $209(98-374)$ & $139(125-266) n=5$ & 0.4274 \\
\hline Before extubation & $255(167-355)$ & $214(118-331) n=3$ & 0.5708 \\
\hline \multicolumn{4}{|l|}{ Glomerular filtration rate (GFR) } \\
\hline Preoperative & $37(28-101)$ & $39(15-114)$ & 0.8531 \\
\hline Postoperative & $34(25-81)$ & $36(22-73)$ & 0.4833 \\
\hline Before TLV & $41(17-97)$ & $38(20-108)$ & 0.5774 \\
\hline 5 days after begin of TLV & $49(22-105)$ & $39(20-62)$ & 0.2811 \\
\hline
\end{tabular}

Frequencies are given for binary data; for quantitative and ordinal data median and range are presented. $p<0.05$ has been considered as statistically significant

overload and intravasal hypervolemia and low serum sodium. We observed that the aquaretic TLV is not only effective in patients with hyponatremia and volume overload due to e.g. cardiac failure as shown earlier, but in especially in small neonates and infants with CLS including massive volume overload in the third space and almost normal sodium levels. In this group the additional aquaresis mobilized the volume from the third space and increase urinary output. In patients with intravasal hypervolemia and low serum sodium, intravascular volume is mobilized. In patients with CLS serum osmolality remains steady, supporting this physiologic hypothesis.

Diverse parameters are discussed to predict the response profiles to TLV however a gold standard has not been established [22, 35, 36]. Especially for our study population of neonates and infants no detailed criteria or predictors for TLV response are known. Thus, one aim of this study was to identify predictors for TLV response in this patient population. In our study cohort we identified urinary output during the first $24 \mathrm{~h}$ and mean BP on day 2 of TLV treatment as good predictors for TLV response. Patients presenting with an increase of urinary output by $1 \mathrm{ml} / \mathrm{kg} / \mathrm{h}$, the probability for TLV response increases by factor 2.1. Further, higher mean BP on day 2 increases the probability of factor 1.2 by each mmHg. Taken together, both parameters represent strong predictors for TLV response.

One potential explanation could be that increased mean $\mathrm{BP}$ at the beginning of TLV therapy in combination with the mechanisms of TLV described above supported and increased TLV effect leading to increased urinary output. On the other side, all other parameters including electrolytes and renal parameters (creatinine, BUN), fluid substitution, transfusions and concomitant medication etc. are not regarded as predictors after multiple regression analysis.

Nevertheless, statements about renal function and GFR are of limited power while using Schwartz formula which is critically discussed as valid parameter for calculating neonatal GFR. Cystatin $C$ which was not routinely measured seems a more predictable parameter to estimate GFR in this patient population. The influence of other potential confounders such as (e.g. PD, adjunctive medication) cannot be completely ruled out, partly due to limited number of patients.

Most likely the response to TLV is also influenced by age, concomitant medication and degree of heart failure. As our study has some limitations because of its retrospective study design and because of the low sample size further studies to identify LTV predictors are necessary.

When comparing responder and non-responder: responder patients presented with significant reduction in body weight and reduction of additional standard diuretic during the TLV treatment course. Further, responder patients showed an improvement of their clinical course by earlier weaning from the ventilator and shorter time on ICU. Nevertheless, these parameters need critical evaluation in a randomized and blinded trial including an untreated control group to validate a positive effect of TLV on outcome parameters. 
In the responder group the main effect of TLV treatment was noticeable during the first 5-6 days. Short-term treatment after cardiovascular surgery might be advantageous compared to long-term treatment due to a discussed TLV escape [13]. In patients who do not show an increase in urinary output (non-responder) a longer treatment should be critically discussed and possibly terminated to reduce potential side effects of TLV in pediatric population.

Side effects of TLV are well described by Otsuka Pharmaceutical and in the literature for adult patients. Nevertheless, pharmacodynamics in children and infants can differ severely from adults and randomized trials are missed in the pediatric population. Despite safety of TLV therapy was not the aim of the study: in our evaluation described side effects were retrospectively analyzed between the two subgroups. TLV was well tolerated particularly in terms of excessive sodium elevations or severe deterioration of liver function which did not occur. We had one case of hypernatremia which was reversible after one day. All patients receiving TLV showed high morbidity and mortality, therefore adverse effects especially on renal and cardiac impairment and long-term outcome could not be evaluated and need further evaluation in a prospective, randomized and blinded trial including an appropriate control group to validate a positive effect of TLV on outcome compared to standard care.

\section{Conclusion}

The use of TLV added to conventional diuretic therapy in infants and neonates after cardiac surgery with CLS was effective in $68 \%$ of our patients with CHD and CLS after cardiac surgery. Responder to TLV presented with increase in urinary output and significant weight reduction. Reduction of diuretic co-medication was possible. Increase in urinary output during $24 \mathrm{~h}$ after TLV treatment and mean BP on day 2 of treatment were strong predictors for TLV response. Prospective, controlled and multicenter studies are desirable and needed to confirm the beneficial effects of TLV and to monitor side effects in the field of pediatric cardiology and neonates.

\section{Additional file}

Additional file 1: Table S1. Preoperative data. Table S2. Vital

parameters. Table S3. Catecholamine therapy, fluid management and transfusion management after surgery. (DOCX $20 \mathrm{~kb}$ )

\section{Abbreviations}

ADH: Antidiuretic hormone; ALT: Alanine Aminotransferease; AST: Aspartate Aminotransferase; AUC: Area under the curve; BP: Blood pressure; BUN: Blood urea nitrogen; CHF: Congestive heart failure; CLS: Capillary leak syndrome; CPB: Cardio pulmonary bypass; CVP: Central venous pressure; FDA: Food and Drug Administration; FFP: Fresh frozen plasma; GFR: Glomerular filtration rate; GGT: Gamma-Glutamyltransferase; HAES: Hydroxyethyl starch;

Hb: Haemoglobin; Hk: Hematocrit; HR: Heart rate; ICU: Intensive care unit;

PCT: Procalcitonin; PRBC: Packed red blood cells; RAAS: Renin-angiotensin- aldosterone-system; SD: Standard deviation; SIADH: Syndrome of Inappropriate Antidiuretic Hormone Secretion; ST-ratio: Subcutaneousthoracic ratio; STS-EACTS: Society of Thoracic Surgeons-European Association for Cardio- Thoracic Surgery; TLV: Tolvaptan; ULN: Upper limit of normal; UV: Urine volume

\section{Acknowledgements}

The presented work was performed in fulfillment of the requirements for obtaining the degree "Dr. med" at "Friedrich-Alexander University of Erlangen-Nürnberg (FAU)" of Anne Kerling. We thank Hakan Toka for critically reviewing the manuscript.

\section{Funding}

None.

Availability of data and materials

The datasets used and/or analysed during the current study are available from the corresponding author on reasonable request.

\section{Authors' contributions}

AK collected and analyzed the data. JM and OT designed the study and interpreted the data. JM and AK drafted the main manuscript. HM and CW performed and interpreted the statistical analyses. SD contributed substantially to the conception and interpretation of the study. AR and SH contributed to the manuscript preparation. All participating authors critically revised the paper before submission. All authors read and approved the final manuscript.

\section{Authors' information}

The Department of Pediatric Cardiology of the Friedrich-Alexander University Erlangen-Nürnberg is a 22 bed unit (including 8 intensive care beds) offering full service for patients with congenital heart disease of all ages and as well for children and adolescents with acquired heart disease. The Department of Pediatric Cardiology treats out about 780 hospital cases including about 420 catheterizations and 230 CPB-surgeries annually.

\section{Ethics approval and consent to participate}

The retrospective study was approved by the ethics committee of the University of Erlangen-Nürnberg (Re.-No. 145_13B). The study was conducted in accordance with the Declaration of Helsinki [32]

Consent for publication

Not applicable.

\section{Competing interests}

The authors declare that they have no competing interests.

\section{Publisher's Note}

Springer Nature remains neutral with regard to jurisdictional claims in published maps and institutional affiliations.

\section{Author details}

${ }^{1}$ Department of Pediatric Cardiology, University of Erlangen-Nürnberg, Loschgestrasse 15,91054 Erlangen, Germany. ${ }^{2}$ Department of Pediatric Cardiac Surgery, University of Erlangen-Nürnberg, Loschgestrasse 15, 91054 Erlangen, Germany. ${ }^{3}$ Department of Pediatrics and Adolescent Medicine, University of Erlangen-Nürnberg, Loschgestrasse 15, 91054 Erlangen, Germany. ${ }^{4}$ Department of Medical Statistics and Biomathematics, University Hospital Mannheim, University of Heidelberg, Theodor-Kutzer-Ufer 1-3, 68167 Mannheim, Germany.

Received: 5 September 2018 Accepted: 28 January 2019

Published online: 12 February 2019

References

1. Lex DJ, Toth R, Czobor NR, Alexander SI, Breuer T, Sapi E, et al. Fluid Overload Is Associated With Higher Mortality and Morbidity in Pediatric Patients Undergoing Cardiac Surgery. Pediatr Crit Care Med. 2016;17(4):307-14.

2. Nicholson GT, Clabby ML, Mahle WT. Is there a benefit to postoperative fluid restriction following infant surgery? Congenit Heart Dis. 2014;9(6):529-35. 
3. Kubicki R, Grohmann J, Siepe M, Benk C, Humburger F, Rensing-Ehl A, Stiller B. Early prediction of capillary leak syndrome in infants after cardiopulmonary bypass. Eur J Cardiothorac Surg. 2013;44(2):275-81.

4. Stiller B, Sonntag J, Dahnert I, Alexi-Meskishvili V, Hetzer R, Fischer T, Lange PE. Capillary leak syndrome in children who undergo cardiopulmonary bypass: clinical outcome in comparison with complement activation and C1 inhibitor. Intensive Care Med. 2001;27(1):193-200.

5. Baehner T, Boehm O, Probst C, Poetzsch B, Hoeft A, Baumgarten G, Knuefermann P. Cardiopulmonary bypass in cardiac surgery. Anaesthesist. 2012;61(10):846-56.

6. Sonntag J, Grunert U, Stover B, Obladen M. The clinical relevance of subcutaneous-thoracic ratio in preterm newborns as a possibility for quantification of capillary leak syndrome. Z Geburtshilfe Neonatol. 2003; 207(6):208-12.

7. Siddall E, Khatri M, Radhakrishnan J. Capillary leak syndrome: etiologies, pathophysiology, and management. Kidney Int. 2017;92(1):37-46.

8. Hassinger AB, Wald EL, Goodman DM. Early postoperative fluid overload precedes acute kidney injury and is associated with higher morbidity in pediatric cardiac surgery patients. Pediatr Crit Care Med. 2014;15(2):131-8.

9. Holmes JH, Connolly NC, Paull DL, Hill ME, Guyton SW, Ziegler SF, Hall RA. Magnitude of the inflammatory response to cardiopulmonary bypass and its relation to adverse clinical outcomes. Inflamm Res. 2002;51(12):579-86.

10. Siehr SL, Shi S, Hao S, Hu Z, Jin B, Hanley F, et al. Exploring the Role of Polycythemia in Patients With Cyanosis After Palliative Congenital Heart Surgery. Pediatr Crit Care Med. 2016;17(3):216-22.

11. Decaux G, Soupart A, Vassart G. Non-peptide arginine-vasopressin antagonists: the vaptans. Lancet. 2008;371(9624):1624-32.

12. Nemerovski C, Hutchinson DJ. Treatment of hypervolemic or euvolemic hyponatremia associated with heart failure, cirrhosis, or the syndrome of inappropriate antidiuretic hormone with tolvaptan: a clinical review. Clin Ther. 2010;32(6):1015-32

13. Boertien WE, Meijer E, de Jong PE, ter Horst GJ, Renken RJ, van der Jagt EJ, et al. Short-term Effects of Tolvaptan in Individuals With Autosomal Dominant Polycystic Kidney Disease at Various Levels of Kidney Function. Am J Kidney Dis. 2015;65(6):833-41.

14. Regen RB, Gonzalez A, Zawodniak K, Leonard D, Quigley R, Barnes AP, Koch JD. Tolvaptan increases serum sodium in pediatric patients with heart failure. Pediatr Cardiol. 2013;34(6):1463-8.

15. Hori M. Tolvaptan for the treatment of hyponatremia and hypervolemia in patients with congestive heart failure. Future Cardiol. 2013;9(2):163-76.

16. Vaduganathan M, Gheorghiade M, Pang PS, Konstam MA, Zannad F, Swedberg K, et al. Efficacy of oral tolvaptan in acute heart failure patients with hypotension and renal impairment. J Cardiovasc Med (Hagerstown). 2012;13(7):415-22.

17. Horibata Y, Murakami T, Niwa K. Effect of the oral vasopressin receptor antagonist tolvaptan on congestive cardiac failure in a child with restrictive cardiomyopathy. Cardiol Young. 2014;24(1):155-7.

18. Kato TS, Ono S, Kajimoto K, Kuwaki K, Yamamoto T, Amano A. Early introduction of tolvaptan after cardiac surgery: a renal sparing strategy in the light of the renal resistive index measured by ultrasound. J Cardiothorac Surg. 2015;10:143.

19. Fukunami M, Matsuzaki M, Hori M, Izumi T, Tolvaptan I. Efficacy and safety of tolvaptan in heart failure patients with sustained volume overload despite the use of conventional diuretics: a phase III open-label study. Cardiovasc Drugs Ther. 2011;25(Suppl 1):S47-56.

20. Higashi K, Murakami T, Ishikawa Y, Itoi T, Ohuchi H, Kodama $Y$, et al. Efficacy and safety of tolvaptan for pediatric patients with congestive heart failure. Multicenter survey in the working group of the Japanese society of PEdiatric circulation and hemodynamics (J-SPECH). Int J Cardiol. 2016;205:37-42.

21. Imamura T, Kinugawa K, Minatsuki S, Muraoka H, Kato N, Inaba T, et al. Tolvaptan can improve clinical course in responders. Int Heart J. 2013; 54(6):377-81.

22. Imamura T, Kinugawa K, Shiga T, Kato N, Muraoka H, Minatsuki S, et al. Novel criteria of urine osmolality effectively predict response to tolvaptan in decompensated heart failure patients--association between non-responders and chronic kidney disease. Circ J. 2013;77(2):397-404.

23. Matsuyama K, Koizumi N, Nishibe T, Iwasaki T, Iwahasi T, Toguchi K, et al. Effects of short-term administration of tolvaptan after open heart surgery. Int J Cardiol. 2016;220:192-5.

24. Kido T, Nishi H, Toda K, Ueno T, Kuratani T, Sakaki M, Takahashi T, Sawa Y. Predictive factors for responders to tolvaptan in fluid management after cardiovascular surgery. Gen Thorac Cardiovasc Surg. 2017;65(2):110-6.
25. Katayama Y, Ozawa T, Shiono N, Masuhara H, Fujii T, Watanabe Y. Safety and effectiveness of tolvaptan for fluid management after pediatric cardiovascular surgery. Gen Thorac Cardiovasc Surg. 2017;65(11):622-6.

26. Jenkins KJ, Gauvreau K, Newburger JW, Spray TL, Moller JH, lezzoni LI. Consensus-based method for risk adjustment for surgery for congenital heart disease. J Thorac Cardiovasc Surg. 2002;123(1):110-8.

27. Boethig D, Jenkins KJ, Hecker $H$, Thies WR, Breymann T. The RACHS-1 risk categories reflect mortality and length of hospital stay in a large German pediatric cardiac surgery population. Eur J Cardiothorac Surg. 2004;26(1):12-7.

28. Lacour-Gayet F, Clarke D, Jacobs J, Comas J, Daebritz S, Daenen W, et al. The Aristotle score: a complexity-adjusted method to evaluate surgical results. Eur J Cardiothorac Surg. 2004;25(6):911-24.

29. Jacobs ML, O'Brien SM, Jacobs JP, Mavroudis C, Lacour-Gayet F, Pasquali SK, et al. An empirically based tool for analyzing morbidity associated with operations for congenital heart disease. J Thorac Cardiovasc Surg. 2013; 145(4):1046-1057.e1.

30. O'Brien SM, Clarke DR, Jacobs JP, Jacobs ML, Lacour-Gayet FG, Pizarro C, et al. An empirically based tool for analyzing mortality associated with congenital heart surgery. J Thorac Cardiovasc Surg. 2009;138(5):1139-53.

31. Imamura $T$, Kinugawa $K$, Minatsuki $S$, Muraoka H, Kato N, Inaba T, et al. Urine osmolality estimated using urine urea nitrogen, sodium and creatinine can effectively predict response to tolvaptan in decompensated heart failure patients. Circ J. 2013;77(5):1208-13.

32. World Medical Association. World Medical Association Declaration of Helsinki: ethical principles for medical research involving human subjects. Jama. 2013;310(20):2191-4

33. Esposito P, Piotti G, Bianzina S, Malul Y, Dal CA. The syndrome of inappropriate antidiuresis: pathophysiology, clinical management and new therapeutic options. Nephron Clin Pract. 2011;119(1):c62-73.

34. Dasta JF, Chiong JR, Christian R, Friend K, Lingohr-Smith M, Lin J, Cassidy IB. Update on tolvaptan for the treatment of hyponatremia. Expert Rev Pharmacoecon Outcomes Res. 2012;12(4):399-410.

35. Kogure T, Jujo K, Hamada K, Saito K, Hagiwara N. Good response to tolvaptan shortens hospitalization in patients with congestive heart failure. Heart Vessels. 2018;33(4):374-83.

36. Shimizu K, Doi K, Imamura T, Noiri E, Yahagi N, Nangaku M, Kinugawa K. Ratio of urine and blood urea nitrogen concentration predicts the response of tolvaptan in congestive heart failure. Nephrology (Carlton). 2015;20(6): 405-12.

Ready to submit your research? Choose BMC and benefit from

- fast, convenient online submission

- thorough peer review by experienced researchers in your field

- rapid publication on acceptance

- support for research data, including large and complex data types

- gold Open Access which fosters wider collaboration and increased citations

- maximum visibility for your research: over $100 \mathrm{M}$ website views per year

At $\mathrm{BMC}$, research is always in progress.

Learn more biomedcentral.com/submissions 